\title{
Status and perspectives of the MEG experiment
}

\author{
Fabrizio $\mathrm{Cei}^{1, \mathrm{a}}$ \\ ${ }^{1}$ INFN and Universitty of Pisa, Department of Physics, Largo B.Pontecorvo 3, 56127 Pisa (Italy) \\ On behalf of the MEG Collaboration
}

\begin{abstract}
We present the status and the perspectives of the MEG experiment which has been searching for the Lepton Flavour Violating decay $\mu^{+} \rightarrow \mathrm{e}^{+} \gamma$ for several years. In a dataset corresponding to $3.6 \times 10^{14}$ positive muons stopped on target we didn't find any evidence for this decay and established an upper bound on the $\mu^{+} \rightarrow \mathrm{e}^{+} \gamma$ branching ratio of $5.7 \times 10^{-13}$ at $90 \%$ C.L., with a sensitivity of $7.7 \times 10^{-13}$, which improves our world best limit by a factor of 4 and the best previous limit by a factor of 20 . Our final dataset is about twice as large than the analyzed sample and the expected final sensitivity of the experiment is $\sim 5 \times 10^{-13}$. We also discuss the status of the upgrade of the experiment (MEGII) which aims to improve the sensitivity by a further order of magnitude.
\end{abstract}

\section{Introduction to Lepton Flavour Violation and $\mu^{+} \rightarrow \mathrm{e}^{+} \gamma$ decay}

The Lepton Flavour Violation (LFV) in the charged lepton sector is almost forbidden in the Standard Model (SM), even including neutrino oscillations and mixing [1]. Conversely, the majority of New Physics models (NP) [2], particularly in view of recent measurements of a large $\theta_{13}$ at reactor [3] and accelerator [4] experiments, predict measurable branching ratios $(\mathcal{B})$ for LFV reactions such as $\mu^{+} \rightarrow \mathrm{e}^{+} \gamma$. The discovery of a LFV process would be clear evidence for NP, whereas improvements in the $\mathcal{B}$ upper limits (UL) constitute significant constraints on the parameter space, complementary to those obtainable at high energy colliders. The expected $\mathcal{B}$ for $\mu^{+} \rightarrow \mathrm{e}^{+} \gamma$ decay ranges from $\sim 10^{-14}$ to $\sim 10^{-12}$; here we show the results and the perspectives of the MEG experiment, which has improved the best world upper limit on this process by a factor of 20 and can reach a sensitivity which covers a large fraction of the allowed parameter space of NP schemes.

\section{The MEG Experiment}

The MEG experiment [5] at Paul Scherrer Institute (PSI) [6] aims to search for the $\mu^{+} \rightarrow \mathrm{e}^{+} \gamma$ decay with a sensitivity $\sim 10^{-13}$. The $\mu^{+} \rightarrow \mathrm{e}^{+} \gamma$ signature for muons decaying at rest is a back-to-back monoenergetic $(52.83 \mathrm{MeV}$ each) time-coincident $\mathrm{e}^{+}-\gamma$ pair. Positron and photon candidates are characterized by their energies $\left(E_{\gamma}, E_{\mathrm{e}}\right)$, their relative directions $\left(\theta_{\mathrm{e} \gamma}, \phi_{\mathrm{e} \gamma}\right)^{1}$ and emission time $\left(t_{\mathrm{e} \gamma}\right)$. The background has two components: the Radiative Muon

\footnotetext{
a e-mail: fabrizio.cei@pi.infn.it

${ }^{1} \theta_{\mathrm{e} \gamma}=\left(\pi-\theta_{\mathrm{e}}\right)-\theta_{\gamma}$ and $\phi_{\mathrm{e} \gamma}=\left(\pi+\phi_{\mathrm{e}}\right)-\phi_{\gamma}, \theta$ and $\phi$ being the polar angle and the azimuthal angle respectively, taking the beam-axis as $z$-axis.
}

Decay $\mu^{+} \rightarrow \mathrm{e}^{+} v_{\mathrm{e}} \bar{v}_{\mu} \gamma(\mathrm{RMD})$, whose rate is proportional to the muon stopping frequency $R_{\mu}$ and the ACCidental Background (ACCB), given by the random coincidence of energetic positrons from the SM Michel decay with photons from RMD, $\mathrm{e}^{+}-\mathrm{e}^{-}$annihilation-in-flight or bremsstrahlung. The ACCB, whose rate is proportional to $R_{\mu}{ }^{2}$, is responsible for $93 \%$ of MEG events with $E_{\gamma}>$ $48 \mathrm{MeV}$.

The MEG experiment uses the PSI $\pi$ E5 beam line which is capable of delivering up to $10^{8}$ stopping $\mu^{+} / \mathrm{s}$ even if a lower intensity of $3 \times 10^{7} \mu^{+} / \mathrm{s}$ is used to control the ACCB. The positive muon beam is stopped in a 205 micron thick plastic target which is slanted $\approx 20^{\circ}$ with respect to the beam axis. The experiment employs a Liquid Xenon (LXe) detector for the measurement of the photon energy, arrival time and first interaction point as well as a magnetic spectrometer for the measurement of the positron momentum vector and timing. The photon detector is formed by a single volume $(\sim 900 \ell)$ of LXe, viewed by 846 UV-sensitive photomultiplier tubes (PMTs) submerged in the liquid. The spectrometer is composed by 16 Drift Chambers (DC), each one formed by two staggered layers of sense wires and cathodic foils, and by a double-array of scintillation Timing Counter (TC) located inside a superconducting solenoid (COnstant Bending RAdius, COBRA) with a gradient magnetic field along the beam axis. The magnetic field is arranged to sweep out positrons with small longitudinal momenta and make the positron bending radius almost independent of emission angle. The detector is continuously monitored by a multi-element calibration system [5, 7, 8] including: 1) a Cockroft-Walton accelerator; 2) an array of $\alpha$ sources mounted inside the photon detector; 3) a liquid hydrogen target, used in conjunction with a $\pi^{-}$beam to induce the Charge Exchange (CEX) reaction $\pi^{-} p \rightarrow \pi^{0} n$, followed 
by the $\pi^{0}$ decay in two photons; 4) a variable monoenergetic positron beam and 5) a neutron generator. The two photons emitted in the CEX calibration are detected in timing and angular coincidence, one in the LXe calorimeter and one in an auxiliary detector, a NaI or a higher resolution BGO array. The relative alignment between the DCs and between the LXe detector and the spectrometer is obtained by looking at straight tracks from cosmic muons and at curved Michel positron tracks and comparing the measurements with an optical survey, improved in 2011 by inserting a laser tracker and prismatic corner cube reflectors mounted on the DC modules. Based on FPGA technology the trigger system is designed to reduce the trigger rate from several $\mathrm{MHz}$ to $\sim 10 \mathrm{~Hz}$ by using fast estimates of photon energy and positron-photon relative timing and direction, with a resulting signal efficiency and live time fraction $>95 \%$ and $\approx 99 \%$ respectively [9]. The readout is performed by a custom-made chip (Domino Ring Sample, DRS), developed at PSI, with a maximum sampling speed of $5 \mathrm{GHz}$ and 12 bit voltage digitization [10].

\section{Data sample}

The experiment collected data continuously and in stable running conditions from 2009 to 2013. A first UL on $\mathcal{B}$ for $\mu^{+} \rightarrow \mathrm{e}^{+} \gamma$ decay was published in 2011 [8] based on the 2009 - 2010 data sample. Here we show the results obtained by analyzing a double-size sample formed by data collected from 2009 to 2011 and corresponding to about $3.6 \times 10^{14}$ positive muons stopped on target.

\section{Analysis procedure and results}

Our analysis is based on a combination of a blind and a maximum likelihood (ML) approach [8], applied in the analysis region defined by $48 \mathrm{MeV}<E_{\gamma}<58 \mathrm{MeV}$, $50 \mathrm{MeV}<E_{\mathrm{e}}<56 \mathrm{MeV},\left|t_{\mathrm{e} \gamma}\right|<0.7 \mathrm{~ns},\left|\theta_{\mathrm{e} \gamma}\right|<50 \mathrm{mrad}$ and $\left|\phi_{\mathrm{e} \gamma}\right|<50 \mathrm{mrad}$. We call "time sidebands" the regions defined by $1 \mathrm{~ns}<\left|t_{\mathrm{e} \gamma}\right|<4 \mathrm{~ns}$, " $E_{\gamma}$-sideband" as defined by $40 \mathrm{MeV}<E_{\gamma}<48 \mathrm{MeV}$ and "angle sidebands" those defined by $50 \mathrm{mrad}<\left|\phi_{\mathrm{e} \gamma}\right|<150 \mathrm{mrad}$ or $50 \mathrm{mrad}<\left|\theta_{\mathrm{e} \gamma}\right|<150 \mathrm{mrad}$. The positron track is reconstructed by combining its measured positions at each DC layer (hit) while longitudinal $z$-positions are derived from signals induced on the segmented DC cathodes. The positron kinematic variables are extracted by using a Kalman filter track fitting technique [11], with a model for the hits and the track based on the GEANE package [12]. An accurate model for multiple scattering and energy loss in the detector materials along with a detailed map of the magnetic field, measured with a $0.2 \%$ precision, are also included. The fitted positron track is propagated to the TC allowing an iterative refinement of the hits with the positron time measurement. The track fit yields a parameter covariance matrix, resulting in very good agreement with the measured resolutions, extracted by using the two turn method [8]. Consequently, a per-track error is determined which allows us to follow the variable DC performance during the data taking period and is taken into account in the ML analysis. The average hit multiplicity for a track is 10 and only tracks with $\geq 7$ hits and $\leq 2$ turns in the spectrometer are retained for the analysis. A single positron per event is selected by applying additional track quality cuts. The positron energy resolution, evaluated by fitting the kinematic edge, is well described by the sum of three Gaussian curves with a resolution $\sigma_{E_{\mathrm{e}}}=(300-310) \mathrm{keV}$ for the core component $(\approx 85 \%)^{2}$. The $\phi_{\mathrm{e}}$-resolution has a $\phi_{\mathrm{e}}$-dependence with a minimum at $\phi_{\mathrm{e}}=0$, where it is measured by the two-turn method to be $\sigma_{\phi_{\mathrm{e}}}=(7-8)$ mrad. Similarly the measured $\theta_{\mathrm{e}}$-resolution is $\sigma_{\theta_{\mathrm{e}}}=(10-11) \mathrm{mrad}$. The decay vertex coordinates and the positron direction at the vertex are determined by extrapolating the reconstructed track back to the target. The resolutions on the decay vertex coordinates, also determined by the two-turn method, are described by a Gaussian curve with $\sigma_{z}=(1.5-2.0) \mathrm{mm}$ and in the vertical direction by the sum of two Gaussian curves with $\sigma_{y}=(1.2-1.3) \mathrm{mm}$ for the core component $(\approx 85 \%)$.

The LXe detector uses the xenon scintillation light to measure the photon position, first interaction time and total energy release. The 3-D photon interaction point is reconstructed by looking at the pattern of scintillation light detected by the PMTs near the incident position and the photon interaction time by combining the leading edge times of the PMT waveforms. The photon direction is defined as the line connecting the decay vertex to the photon interaction point in the LXe detector. The photon energy reconstruction is based on the sum of the charges collected by all PMTs. Monochromatic $55 \mathrm{MeV}$ photons from $\pi^{0}$ decay are used to determine the absolute energy scale. Pile-up photons, which at $R_{\mu}=3 \times 10^{7} \mu^{+} / \mathrm{s}$ affect $\approx 15 \%$ of triggered events, are identified topologically by the PMT light distribution, temporally by the PMT leading edge time distribution and by means of a new algorithm which analyzes waveforms after summing all channels at the end of the full chain of photon reconstruction. This algorithm also allows to re-adjust the charge integration window for the energy estimate, resulting in a better energy reconstruction. The efficiency of photon reconstruction, obtained by comparing CEX data with MC simulations, is 63\%, with a $4 \%$ improvement due to the new algorithm mentioned above. The position reconstruction resolution, evaluated by a MC simulation and validated in CEX runs by placing lead slit collimators in front of the LXe detector, is $5 \mathrm{~mm}$ on the photon entrance face and $6 \mathrm{~mm}$ along the radial depth $w$. The timing and energy resolutions are evaluated using 55 and $83 \mathrm{MeV}$ photons from $\pi^{0}$ decay. The LXe timing resolution is $\sigma_{t_{\gamma}}=67 \mathrm{ps}$ at $52.8 \mathrm{MeV}$. The position-dependent energy resolutions are measured in the CEX data and the average energy resolution, extracted from a Gaussian fit to the high energy side of the spectrum, results $1.7 \%$ and $2.4 \%$ for $w>2 \mathrm{~cm}$ and $w<2 \mathrm{~cm}$ respectively. The resolutions of the relative directions are obtained by combining the relevant resolutions of positrons and photons discussed above. The results are $\approx 16 \mathrm{mrad}$ for $\theta_{\mathrm{e} \gamma}$ and $\approx 9 \mathrm{mrad}$ for

\footnotetext{
${ }^{2}$ The ranges here and after take into account the small, yearly differences caused by various factors (e.g. variable DC configurations and efficiencies).
} 

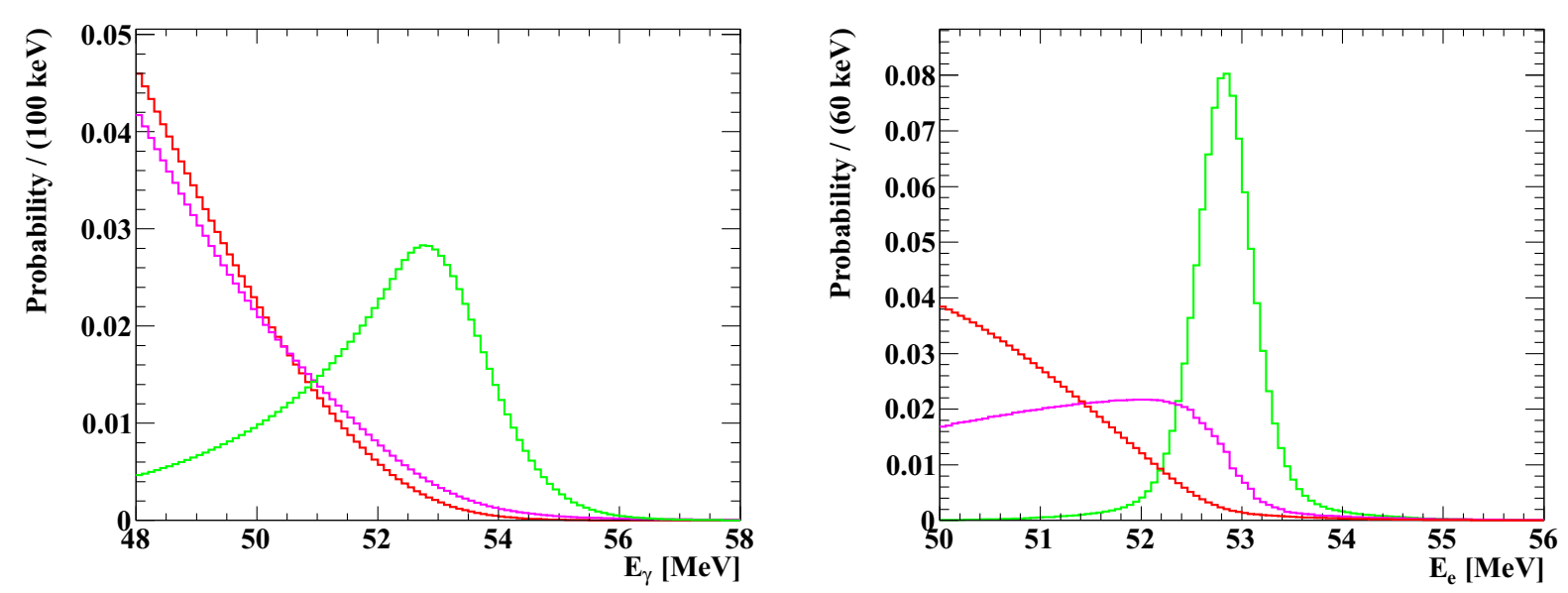

Figure 1. PDF for photon energy (left) and for positron energy (right). In both figures green curves are for signal, red curves for RMD and purple curves for ACCB.
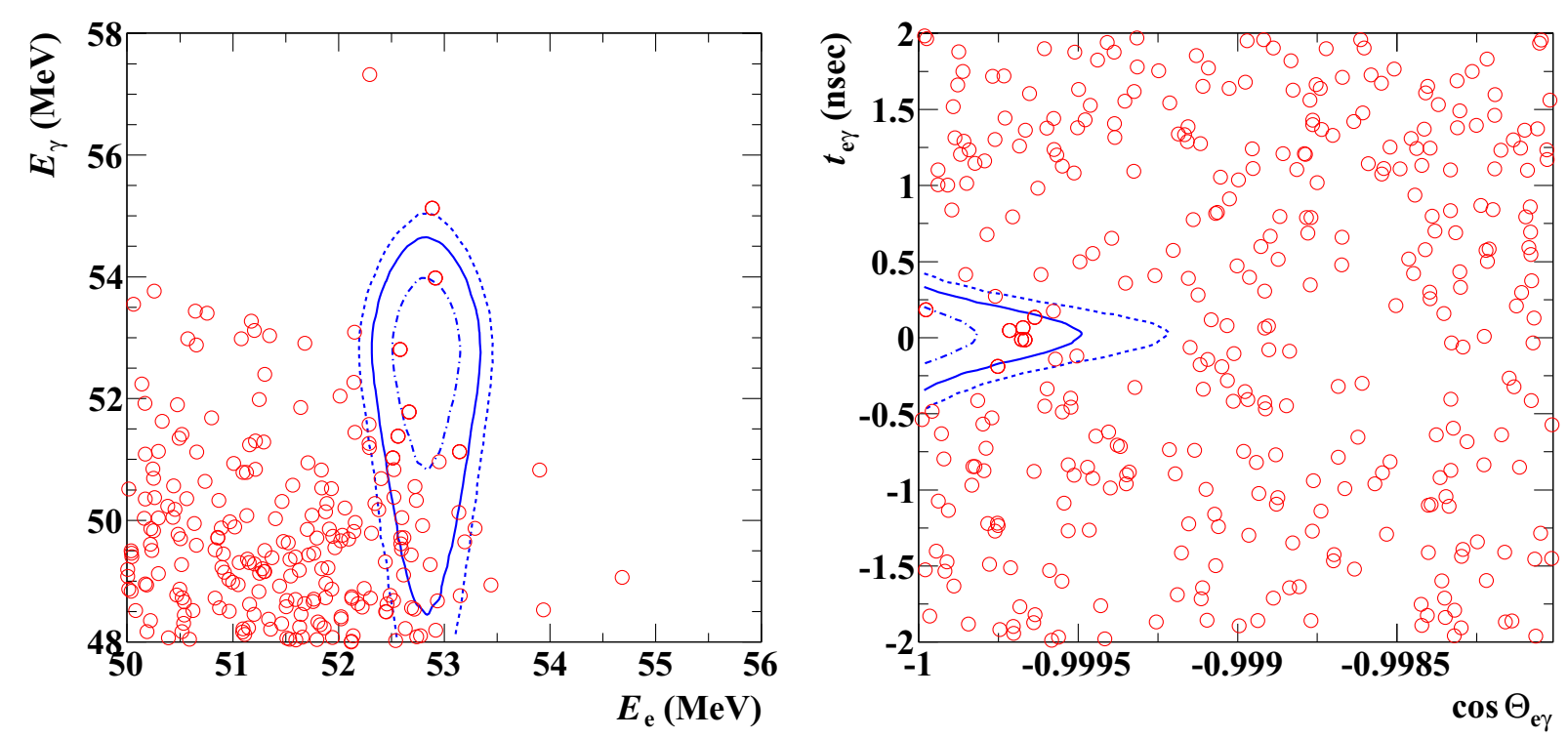

Figure 2. Event distributions for the $2009-2011$ dataset in the $\left(E_{\mathrm{e}}, E_{\gamma}\right)$ and $\left(\cos \Theta_{\mathrm{e} \gamma}, t_{\mathrm{e} \gamma}\right)$ planes. In the figure on the left (right) a selection of $\left|t_{\mathrm{e} \gamma}\right|<0.244 \mathrm{~ns}$ and $\cos \Theta_{\mathrm{e} \gamma}<-0.9996$ with $90 \%$ efficiency for each variable $\left(52.4 \mathrm{MeV}<E_{\mathrm{e}}<55 \mathrm{MeV}\right.$ and $51 \mathrm{MeV}<$ $E_{\gamma}<55.5 \mathrm{MeV}$ with $90 \%$ and $74 \%$ efficiencies for $E_{\mathrm{e}}$ and $E_{\gamma}$, respectively) is applied. The signal PDF contours $(1,1.64$ and $2 \sigma)$ are also shown.

$\phi_{\mathrm{e} \gamma}$. The relative time $t_{\mathrm{e} \gamma}$ is derived from the time measurements in the LXe detector and in the TC, after correcting for the particle time-of-flights. The corresponding resolution at $52.8 \mathrm{MeV}$ energy is $\approx 130 \mathrm{ps}$, measured from the RMD peak observed in the $E_{\gamma}$-sideband above the flat ACCB distribution; a small correction takes into account the $E_{\gamma}$-dependence measured in the CEX runs. The position of the RMD-peak $\left(t_{\mathrm{e} \gamma}=0\right)$, monitored constantly during the data taking period, is stable within $15 \mathrm{ps}$.

The ML analysis is supplemented by a blind analysis procedure which involves masking a region of $48 \mathrm{MeV}<$ $E_{\gamma}<58 \mathrm{MeV}$ and $\left|t_{\mathrm{e} \gamma}\right|<1 \mathrm{~ns}$ until the Probability Density Functions (PDFs) for the likelihood function are finalized. The time, energy and angle sidebands are used to optimize the analysis, to study ACCB and to extract the corresponding PDFs. Calibration data and measured resolutions are used to build the Signal (S) and RMD events PDFs. Different resolutions and correlations are included in the PDFs on an event-by-event basis. The per-event positron error matrix, estimated by the new Kalman filter, is introduced in the PDFs, together with the dependence on the photon interaction point and the quality of the positron tracking. Examples of PDFs for photon and positron energy are shown in Fig. 1.

All the PDFs are inserted in the global likelihood function [8] which includes also the sideband constraints on the number of RMD and ACCB events. The ML fit is performed to estimate the number of S, RMD and ACCB 

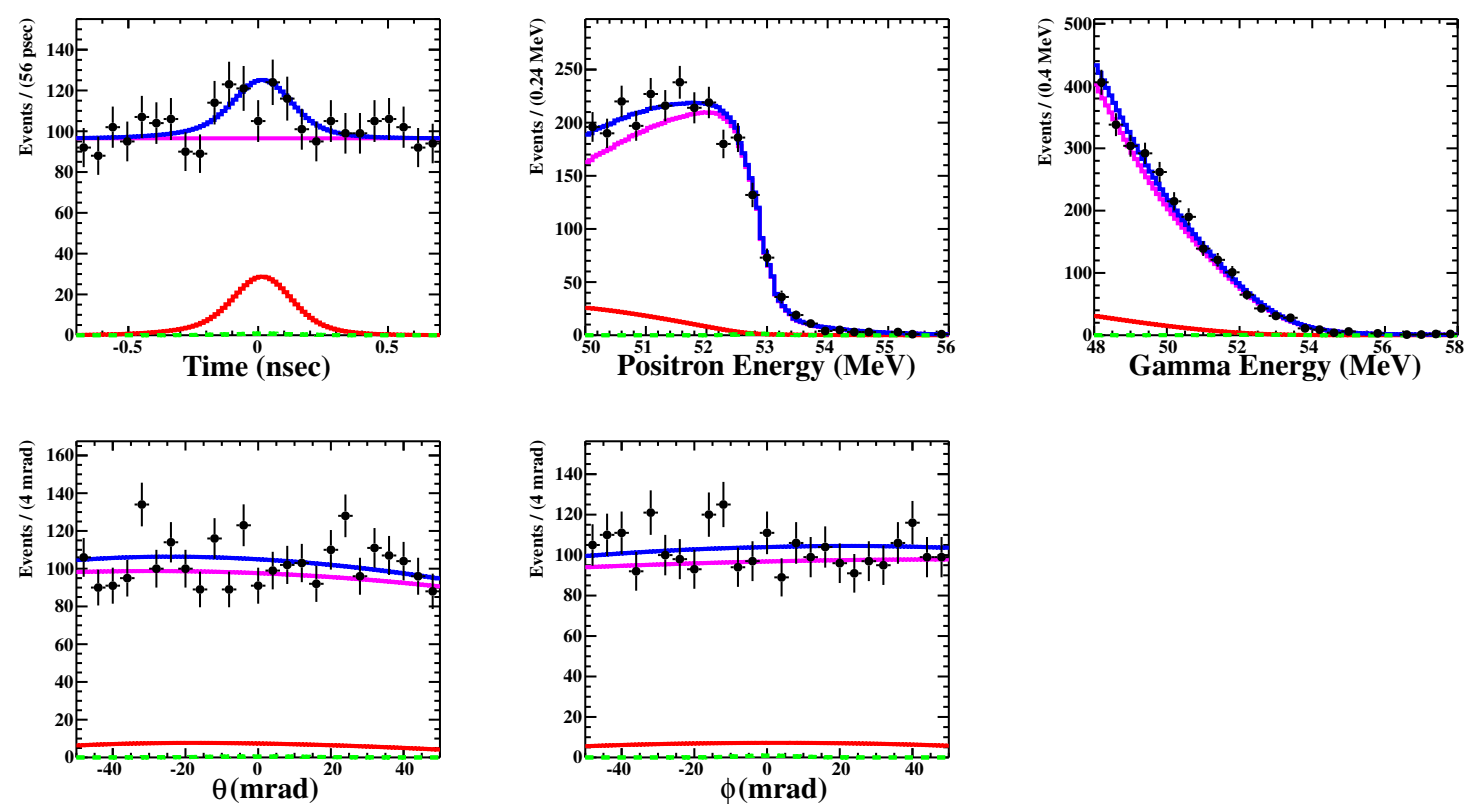

Figure 3. Results of ML fit for the 2009 - 2011 dataset. The black dots are the data and the colour lines represent the contributions extracted from the fit: S (green), RMD (red), ACCB (purple) and total (blue).

events in the analysis region. An analysis with constant PDFs is also performed as a crosscheck and has shown consistent results. The confidence interval for the number of $\mathrm{S}$ events is calculated by a frequentist method with a profile likelihood-ratio ordering $[8,13]$, where the numbers of RMD and ACCB events are treated as nuisance parameters. The experimental sensitivity $\mathcal{S}_{90}$ is evaluated as follows: a large sample of pseudo experiments (toy MCs) with 0 signal events is generated and fit with the ML algorithm. $\mathcal{S}_{90}$ is defined as the median of the distribution of the $90 \%$ C.L. ULs extracted from the toy MC ensemble. Likelihood fits are also performed in fictitious analysis regions in the time and angle sidebands, getting ULs in good agreement with the $\mathcal{S}_{90}$ 's. The normalization factor needed to convert a UL on the number of $\mathrm{S}$ events into a UL on $\mathcal{B}\left(\mu^{+} \rightarrow \mathrm{e}^{+} \gamma\right)$ is computed by using two independent schemes; either counting the number of Michel positrons selected with a dedicated pre-scaled trigger, or the number of RMD events observed in the muon data. The combined uncertainty on the $\mathcal{B}$ value is $4 \%$. The systematic uncertainties on the PDF parameters and on the normalization are taken into account in the calculation of the confidence intervals by fluctuating the PDFs by the amount of the uncertainties. The global effect on the observed UL is $1 \%$ with the main contribution coming from the angular PDFs. Fig. 2 shows the event distributions in the $\left(E_{\mathrm{e}}, E_{\gamma}\right)$ and $\left(\cos \Theta_{\mathrm{e} \gamma}, t_{\mathrm{e} \gamma}\right)$ planes for the 2009 - 2011 dataset, where $\Theta_{\mathrm{e} \gamma}$ is the opening angle between positron and photon, together with the contours of the averaged signal PDFs. We show in Fig. 3 the results of the ML fit for the dataset 2009 - 2011 where the best fit value for the number of $\mathrm{S}$ events is -0.4 . The observed profile likelihood ratios as a function of $\mathcal{B}$ are shown in
Fig. 4 for three different datasets: $2009-2010,2011$ and 2009 - 2011 combined sample. The UL at $90 \%$ C.L.

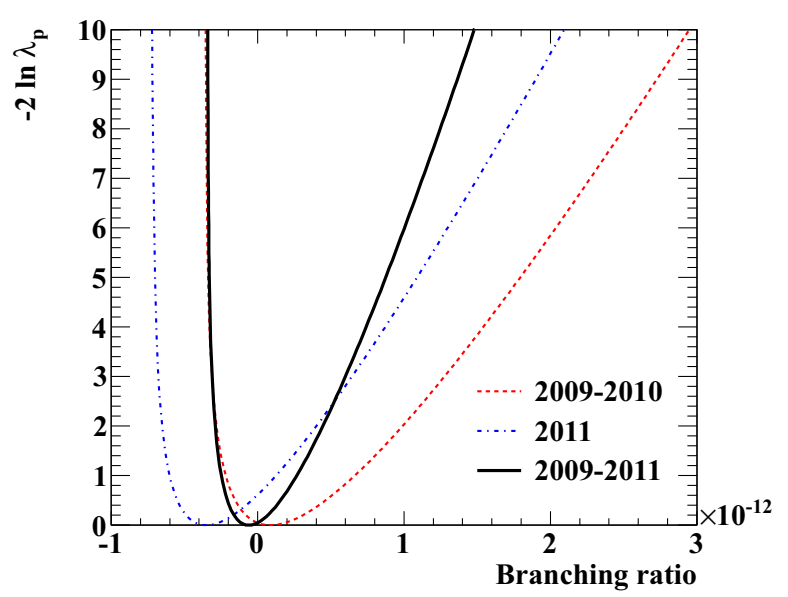

Figure 4. Observed profile likelihood ratios $\left(\lambda_{\mathrm{p}}\right)$ as a function of $\mathcal{B}$ for 3 different data sets: $2009-2010,2011$ and the combined 2009 - 2011 sample.

$\left(\mathcal{B}_{90}\right)$ and $\mathcal{S}_{90}$ for the full $2009-2011$ dataset are respectively $5.7 \times 10^{-13}$ and $7.7 \times 10^{-13}$. As a quality check the ML fit was repeated omitting the constraint on the number of background events. We obtained $N_{\mathrm{RMD}}=163$ \pm 32 and $N_{\mathrm{ACCB}}=2411 \pm 57$, in good agreement with the expectations estimated from $E_{\gamma}$ and time sidebands $\left\langle N_{\mathrm{RMD}}\right\rangle=169 \pm 17$ and $\left\langle N_{\mathrm{ACCB}}\right\rangle=2415 \pm 25$. The obtained UL is more stringent than $\mathcal{S}_{90}$, but this is not unusual since the probability of having $\mathcal{B}_{90}$ equal or smaller than the observed quantity is calculated to be $24 \%$ with a 


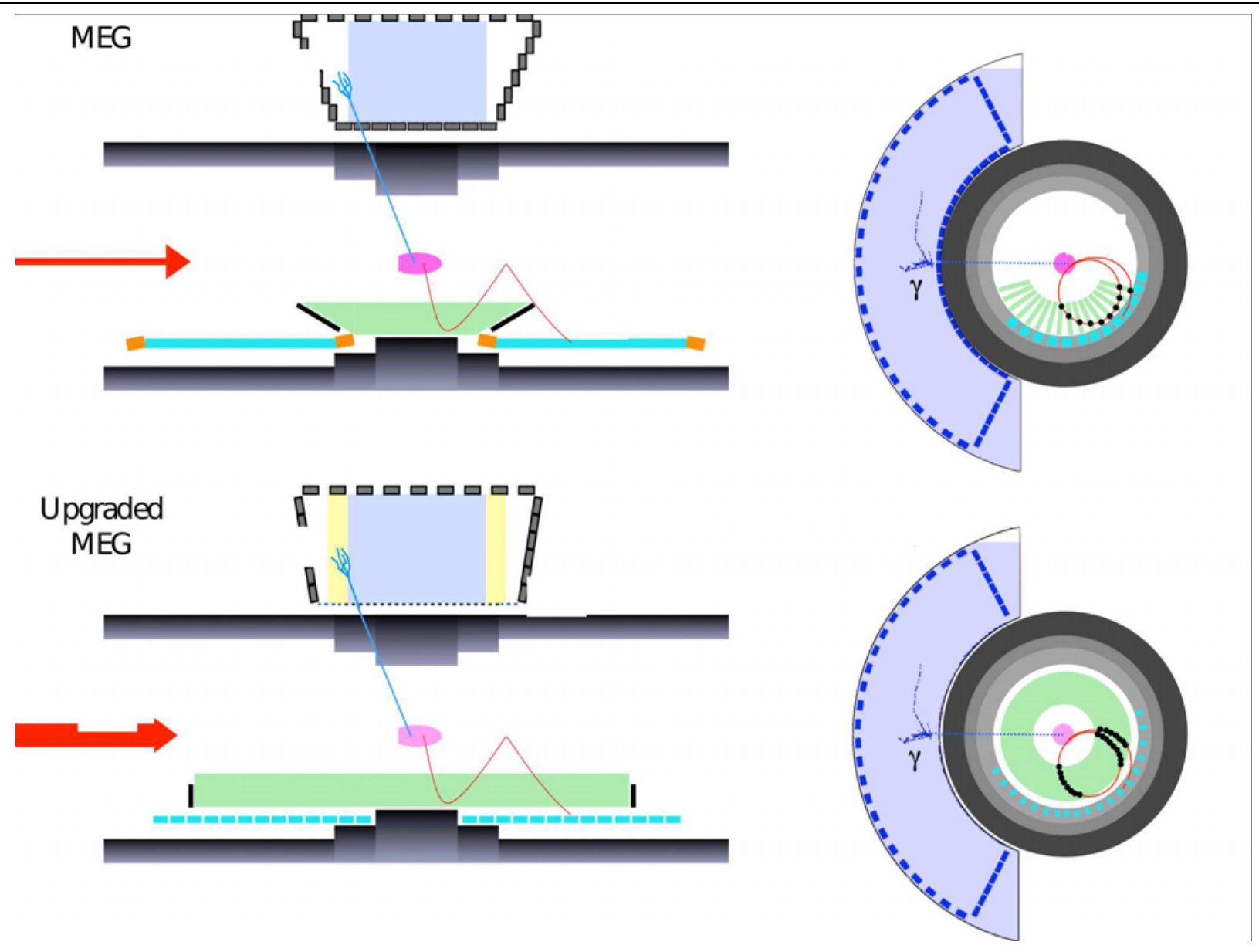

Figure 5. Overview of the MEG upgrade compared with the present MEG configuration.

sample of toy MC experiments. These results were published in 2013 [14] and improve the previous MEG UL [8] by a factor of 4 .

\section{Expected final sensitivity}

The results were obtained by analyzing a data sample corresponding to about half of the total amount of data collected by MEG with the total number of positive muons stopped on target about $7.2 \times 10^{14}$. The analysis of the remaining data is in progress and is expected to be completed shortly. The present Upper Limit $\mathcal{B}\left(\mu^{+} \rightarrow \mathrm{e}^{+} \gamma\right)<$ $5.7 \times 10^{-13}$ at $90 \%$ C.L. represents an improvement by a factor of 20 with respect to the pre-MEG limit [15] and a significant constraint for several NP models. When the full statistics will be analyzed we expect to reach a final sensitivity, estimated by using toy MC's, of $\sim 5 \times 10^{-13}$. It's important to note that, despite the expected doubling of data statistics, the predicted improvement in sensitivity is only of about a factor of 1.5. This is due to the fact that systematic effects dominate the limiting sensitivity because of the presence of an irreducible background. The unique way to obtain a much more significant improvement, at the level of a further order of magnitude in sensitivity, is to reduce the spill-in of background events within the signal region by enhancing the detector resolution. This was the basic motivation for the MEG upgrade project.

\section{The MEG upgrade}

The MEG upgrade [16] project was based on the long-term experience we gained during MEG phase I. The guideline was the possibility to take advantage of a higher intensity beam (from $3 \times 10^{7}$ to $7 \times 10^{7}$ stopping positive muons per second), thanks to an enlarged detector acceptance and to better resolution sub-detectors, to be obtained with moderate costs and on a relatively short time-scale $(\sim 3-4$ years). An overview of the MEG upgrade is shown in Fig. 5.

The main element of the upgrade is the replacement of the present DC system with a $2 \mathrm{~m}$ long unique volume cylindrical drift chamber. With respect to the present MEG configuration, this new device has 1) a higher momentum, 2) a higher angular resolution and 3) a much 
Table 1. Resolution (Gaussian $\sigma$ ) and efficiencies for MEG upgrade

\begin{tabular}{lll}
\hline PDF parameters & Present MEG & Upgrade scenario \\
\hline$\sigma_{E_{e^{+}}}(\mathrm{keV})$ & 380 & 110 \\
$e^{+} \sigma_{\theta}(\mathrm{mrad})$ & 11 & 5 \\
$e^{+} \sigma_{\phi}(\mathrm{mrad})$ & 9 & 5 \\
$e^{+} \sigma_{Z} / \sigma_{Y}(\mathrm{core})(\mathrm{mm})$ & $2.0 / 1.2$ & $1.2 / 0.7$ \\
$\frac{\sigma_{E_{\gamma}}}{E_{\gamma}}(\%) w>2 \mathrm{~cm}$ & 1.7 & 1.0 \\
$\gamma$ position at LXe $\sigma_{(u, v)}-\sigma_{w}(\mathrm{~mm})$ & 5 & 2 \\
$\gamma-e^{+}$timing (ps) & 130 & 80 \\
\hline Efficiency (\%) & & \\
\hline trigger & $\approx 99$ & $\approx 99$ \\
$\gamma$ reconstruction & 63 & 63 \\
$e^{+}$reconstruction & 40 & 95 \\
event selection & 80 & 85 \\
\hline
\end{tabular}

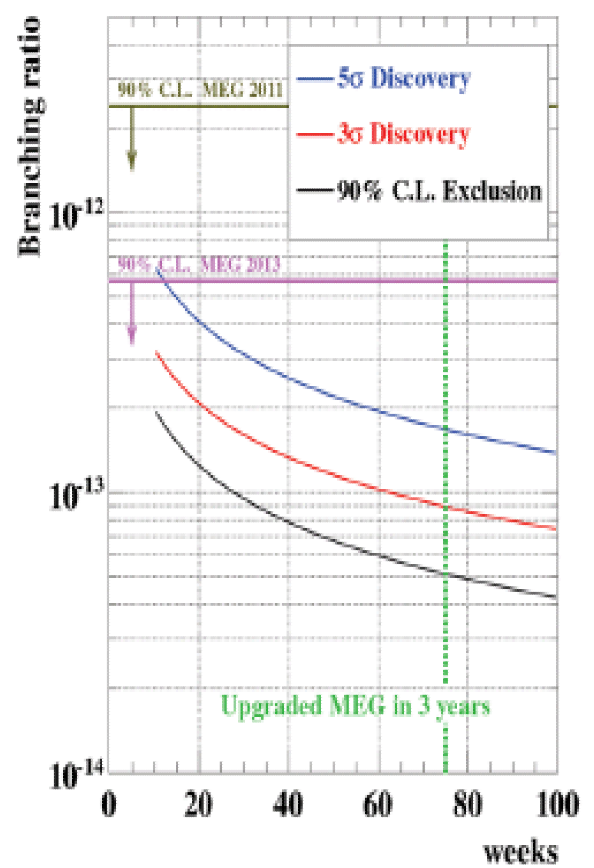

Figure 6. Expected sensitivity of MEG upgrade as a function of DAQ time. The present [14] and previous [8] MEG upper limits are indicated by arrows. The Blue, Red and Black curves show respectively the $\mathcal{B}$ corresponding to a discovery at $5 \sigma$ and $3 \sigma$ levels and the $90 \%$ C.L. exclusion line.

larger positron efficiency because of the reduction of the material budget traversed by the positron and of the much better coupling with the TC. Moreover, since no cathode foils will be used for the $z$ coordinate reconstruction, there will be no problems of low level signals and of electric discharges due to the formation of charge deposits on the cathodes. The new DC will be equipped with $\sim 1400$ sense wires and $\sim 8000$ field and guard wires with a $2 \pi$ azimuthal coverage and will be operated with a $85: 15$
$\mathrm{He} /$ Isobutane gas mixture. The amount of traversed material corresponds to less than $2 \times 10^{-3} X_{0}$ and the stereo view angle is $(7-8)^{\circ}$. The single hit resolution is expected to be 120 micron. This value was tested with laboratory measurements on a small and a full length prototype by using cosmic rays and beam tests [17]. The small prototype was also used to check that the expected ageing of the gas mixture during the MEG II DAQ time will produce a maximum gain drop of $40 \%$, which can be cor- 
rected by increasing the $\mathrm{HV}$, and that the $z$ coordinate can be reconstructed with $\mathrm{a} \sim 10 \mathrm{~cm}$ precision by using the charge division on the two sides of signal wires. Other major upgrades involve:

a) a different target configuration, with 140 micron thickness and a slant angle of $15^{\circ}$, to better substain the increased muon stopping rate.

b) The replacement of LXe photon detector inner face PMTs with an array of $12 \mathrm{~mm} \times 12 \mathrm{~mm}$ Silicon Photomultipliers (SiPM) sensitive to LXe scintillation light. The increased granularity and active coverage are expected to enhance 1) the light collection efficiency, 1) the uniformity and 3) the pile-up rejection capability with an improvement of a factor of 2 in energy and position resolution that is considerable for shallow events where the photon converts very close to the entering thin window. The photon detection efficiency is also expected to increase by some percent because of the smaller mass to be traversed by the photon entering the LXe detector. About 600 SiPMs were already tested and show a good photon detection efficiency for LXe scintillation light.

c) A redesign of the LXe detector lateral sides and a better suited placement of PMTs on these sides to improve the acceptance and the light collection uniformity.

d) The building of a new pixellated Timing Counter, made by a 2-D array of scintillator tiles, to improve the resolution of positron timing measurement by means of multiple hits in different tiles. The single tile resolution and the dependence of the timing resolution as a function of the number of crossed tiles were measured in beam tests and helped obtain an average positron timing resolution of $\approx 35 \mathrm{ps}$, in agreement with upgrade project needs.

e) The building of a new mixed trigger/digitizer DAQ board (WaveDREAM) in order to fulfill the requests of an increased number of read-out channels and of a higher bandwidth of the analog Front-End.

f) The insertion in the DownStream side of the experiment of a LYSO+plastic scintillator detector to detect low energy positrons emitted in RMD decay and tag the corresponding high-energy photon coming from the same decay. This detector is already under construction.

The figures of merit of the upgraded detector are compared in Table 1 with that of the present detector. The expected sensitivity, the $3-\sigma$ and the $5-\sigma$ discovery curves as a function of the DAQ time are shown in Fig. 6. With 3 years of data taking we expect to reach a sensitivity of $\sim 5 \times 10^{-14}$ with an improvement of one order of magnitude with respect to the estimated final sensitivity of MEG phase I.

\section{References}

[1] F. Böhm \& P. Vogel, Physics of Massive Neutrinos, Cambridge University Press, (1992), S. T. Petcov, Sov. J. Nucl. Phys. 25 (1977) 340.

[2] R. Barbieri, L. Hall and A. Strumia, Nucl. Phys. $B 455$ (1995) 219, J. Hisano, D. Nomura and T. Yanagida, Phys. Lett. B 437 (1998) 351, M. Raidal et al., Eur. Phys. J. C 57 (2008) 13, G. Blankenburg et al., Eur. Phys. J. C 72 (2012) 2126.

[3] F. P. An et al., Phys. Rev. Lett. 108 (2012) 171803, F. P. An et al., Phys. Rev. Lett. 112 (2014) 061801, J. K. Ahn et al., Phys. Rev. Lett. 108 (2012) 191802, Y. Abe et al., Phys. Lett. B 723 (2013) 66.

[4] K. Abe et al., Phys. Rev. Lett. 107 (2011) 041801, K. Abe et al., Phys. Rev. Lett. 112 (2014) 061802.

[5] J. Adam et al., Eur. Phys. J. C 73 (2013) 2365

[6] http://www.psi.ch

[7] J. Adam et al., Nucl. Instr. Meth. A 641 (2011) 19, A. M. Baldini et al., Nucl. Instr. Meth. A 565 (2006) 589, A. M. Baldini et al., Nucl. Instr. Meth. A 545 (2006) 753

[8] J. Adam et al., Phys. Rev. Lett. 107 (2011) 171801

[9] L. Galli et al., JINST 8 (2013) P01008.

[10] S. Ritt et al., Nucl. Instr. Meth. A 623 (2010) 486

[11] P. Billoir, Nucl. Instr. Meth. A 225 (1984) 352, R. Frühwirth, Nucl. Instr. Meth. A 262 (1987) 444.

[12] V. Innocente and E. Nagy, Nucl. Instr. Meth. A 324 (1993) 297, A. Fontana et al., J. Phys. Conf. Ser. 119 (2008) 032018.

[13] J. Beringer et al. (Particle Data Group), Phys. Rev. D 86 (2012) 010001, G. J. Feldman and R.D. Cousins, Phys. Rev. D 57 (1998) 3873.

[14] J. Adam et al., Phys. Rev. Lett. 110 (2013) 201801

[15] M. Ahmed et al., Phys. Rev. D. 65 (2002) 112002.

[16] A. M. Baldini et al., MEG Upgrade Proposal, physics.ins-det/1301.7225.

[17] M. Venturini et al., Nucl. Instr. Meth. A (2015) doi:10.1016/j.nima.2015.09.063. 